\title{
Comparative Study between COVID Associated Guillan Barre Syndrome and Non-COVID Associated Guillan-Barré Syndrome
}

\author{
Kanika Taneja', Kalpana Zutshi ${ }^{2}$, Loveneet Bharti $^{3}$ \\ ${ }^{1}$ Consultant Physiotherapist, ${ }^{3}$ Physiotherapist Intern, Sir Ganga Ram Hospital, BPT, Sharda University, New Delhi, India. \\ ${ }^{2}$ Associate Professor, Department of Rehabilitation Sciences, SNSAH, Jamia Hamdard, New Delhi, India. \\ DOI: https://doi.org/10.24321/2394.6539.202110
}

I $\quad \begin{array}{lllll}\mathbf{N} & \mathbf{F} & \mathbf{O}\end{array}$

Corresponding author:

Kalpana Zutshi, Department of Rehabilitation Sciences, SNSAH, Jamia Hamdard,New Delhi, India.

E-mail Id:

zutshi.kalpana@gmail.com

Orcid Id:

https://orcid.org/0000-0002-3270-8068

How to cite this article:

Taneja K, Zutshi K, Bharti L. Comparative Study between COVID Associated Guillan Barre Syndrome and Non-COVID Associated GuillanBarré Syndrome. J Adv Res Med Sci Tech. 2021;8(3):10-12.

Date of Submission: 2021-04-15

Date of Acceptance: 2021-09-10 $\begin{array}{llllllllllllllll}\text { I } & \mathbf{N} & \mathbf{T} & \mathbf{R} & \mathbf{O} & \mathbf{D} & \mathbf{U} & \mathbf{C} & \mathbf{T} & \mathbf{I} & \mathbf{O} & \mathbf{N}\end{array}$

More than 12 million people have been infected with COVID-19 worldwide, with more than 500,000 deaths to date. ${ }^{1}$ Although COVID-19 research is rapidly evolving, new findings must be thoroughly scrutinised before any conclusions or treatment protocols are established or amended. ${ }^{2}$ Although COVID-19 is most usually associated with respiratory symptoms such as cough and dyspnea; it has recently been associated with a neurotropic presentation. ${ }^{3}$ Guillain-Barré Syndrome (GBS) is best described as an acute inflammatory polyradiculoneuropathy clinically characterized by areflexia and progressive weakness of arms and legs. Though, many rare variants of GBS have been described, the commonly observed subtypes such as Acute Motor Axonal Neuropathy (AMAN), Acute Motor Sensory Axonal Neuropathy (AMSAN) and Acute Inflammatory Demyelinating Polyradiculoneuropathy (AIDP) tend to fulfil the above-mentioned criteria. ${ }^{4}$ Recently, several case reports have suggested a relationship between the occurrence of Guillain-Barré syndrome (GBS) and a previous SARS-CoV-2 infection, which preceded the GBS onset by up to 4 weeks. Therefore, a postinfectious dysregulation of the immune system, triggered by SARSCoV2, appears to be the most probable cause COVID-19 is a systemic disorder presenting typically with fever and respiratory symptoms but neurological manifestations such as acute cerebrovascular diseases, seizures, ageusia, anosmia meningitis, encephalitis and skeletal muscle involvement were soon reported. ${ }^{5}$ More recently, an increase in case reports of Guillain-Barré syndrome (GBS) in people infected with SARSCoV-2 has prompted concerns about a possible link.

\section{Case Study I}

COVID Negative with Guillain-Barré Syndrome Without Respiratory

Involvement

A 52 year old male came to outpatient department of nearby tertiary care centre with chief complaints of low-back pain radiating to both lower limbs. 
Patient presented with clinical features of pain in back and going to both legs and also feeling of weakness. He also suffered with facial muscle deviation. He was suggested to proceed for Magnetic Resonance Imaging (MRI).

MRI spine findings suggesting of partial sacralization of $\mathrm{L} 5$ vertebra with concentric disc bulge, ligamentum flavum thickening and facet effusion at L4-L5 level causing compression of bilateral transversing nerve roots. And MRI brain said no acute infarct.

He had a history of fever and cough two weeks back and which had settled in 6 to 7 days. At that time, when he was tested for COVID, it was negative. After 4 days, he came to our hospital with chief complaints of bifacial palsy with paresthesia in lower limbs and he was not able to stand.

His nerve conduction study was conducted for both upper limb and lower limb and results showing distal symmetrical sensorimotor neuropathy involving bilateral median nerve possibly due to carpal tunnel syndrome (right severe, left moderate) and normal distal latency, amplitude and conduction velocity in bilateral ulnar, common peroneal and posterior tibial nerves. $\mathrm{F}$ response was absent in bilateral median nerve and $\mathrm{H}$ reflex was absent bilaterally. He was treated with IV Immunoglobulin, and physiotherapy intervention was given; other investigations were $\mathrm{Hb}-16.4 \mathrm{~g} /$ dl , TLC- 9.85 , ESR- , PCV 48.3\%, MCV-81.3fl, MCHC- 34g/dl MCH-27.6 pg, RDW-15.6\%, DLC-lymphocyte 34\%, monocyte $6 \%$, D-dimer-200 ng/dl, ferritin-150 ng/ml. On RFT only sodium was little less $131.00 \mathrm{mmol} / \mathrm{l}$ rest all was normal, creatinine $0.81 \mathrm{mg} / \mathrm{dl}$. CPK was $202 \mathrm{IU} / \mathrm{I}$. 2D Echo- LVEF$60.2 \%$, RBS- $150 \mathrm{mg} / \mathrm{dl}$.

\section{Case Study 2}

\section{COVID Positive with Guillain-Barré Syndrome with Respiratory Involvement}

A 52 year old male came in an outpatient department of tertiary care centre with chief complaints of difficulty in walking, throat congestion, back pain, numbness and burning in feet, loss of appetite and difficulty in swallowing for last 3 days. Patient was diagnosed as COVID 19 positive by RT-PCR test with paraplegia with chest infection and then he got admitted to COVID ward urgently. He was hypertensive and on medications.

On investigation, ESR - 45mm 1st hr, Haemoglobin 11.6g/dl, PCV 37.5\%, MCV 80.5fl, MCHb 24.9pg, MCHb 30.9\%, RDW 49.8fl, RDW 17.2\%, DLC lymphocyte $18 \%$, monocyte $12 \%$, D-dimer $422.9 \mathrm{ng} / \mathrm{dl}$, creatinine $0.7 \mathrm{mg} / \mathrm{dl}$. He was started on medications Inj. Sulbitor, Tab Ecosporin, Tab CCM, Tab Arkamin, Tab Amlovas, Inhaler Duolin and budecort, Inj PAN , Tab LIMCEE, Tab Zincovit, betadine gangles, Inj doxy, Tab montair LC, Tab lorfast AM, Inj Remdesivir, Tab ivermectin for 2 days, then on third day his attendants asked doctors to transfer the patient to another hospital. On the day of discharge, he were examined, BP 150/100 mmHg, PR 110b/ $\mathrm{min}, \mathrm{RR} 20 / \mathrm{min}, \mathrm{SPO} 2$ 95\%, CNS - conscious, oriented, chest - bilateral air entry, CVS-S1S2 present, P/A - soft, non-tender. His other reports were also reviewed with investigations of CBC-Hb 13.1, TLC 7800, ESR20, platelet 166000 , KFT urea 22, creatinine 0.33 , procal 0.03, IL6 6.60, D-Dimer 422.9,Trop 1.80, pro BNP 91.0, serum ferritin 264.78, 2D Echo LVEF 60\% , RBS 172 mg/dl. CT head was done suggestive of normal study. He got transferred to other tertiary care center.

His bedside nerve conduction study was conducted in right upper limb and both lower limb. Nerve conduction study showing distal symmetrical large fiber sensorimotor mixed axonal + demyelinating polyneuropathy/polyradiculopathy involving both lower limb and upper limb. Sural nerve was spared. His MMT was $3 / 5$ proximal upper limb and $1 / 5$ lower limb proximal and 2/5 distal.

\section{Treatment Received}

\section{For Case I}

He was admitted to tertiary care centre, initially his medical treatment involved injection clexane and inj perfalgan and was started on inj. Globucel (10 g). The patient was treated with intravenous immune globulin (IVIG).

Physiotherapy management involved limb and chest exercise programme which included active assisted range of motion exercises of all four limbs along with proprioception training. Chest programme included deep breathing exercises, spirometry; Active Cycle of Breathing Technique (ACBT) consisted of breathing control, thoracic expansion exercises, huffing and coughing technique for 7 days and after that strengthening exercises were started along with ambulation.

\section{For Case 2}

Patient had been tracheostomised and got treated with inj. remdesvir and inj. Doxy , IV antibiotics, inhaler duolin and budecort. The patient received a second course of IVIG and started plasma exchange as well.

Along with it, regular chest and limb physiotherapy involving percussions, vibration, shaking, body positioning, active assisted ROM exercises of both upper and lower limbs, progressive resistive training. Patients have shown improvement by above-mentioned interventions and need further rehabilitation for motor control and other intervention such as gait training, biofeedback training, functional training, proprioception training(proprioceptive neuromuscular facilitation), speech therapy, coordination impairments, muscle power and endurance, strength, aerobic training, flexibility exercises, postural control and balance, agility training, relaxation training and rest according to the condition. 


\section{Follow Up Care \& Advices}

\section{Case I}

Patient was asked to come for follow up every week and was continued on immunoglobolins along with physiotherapy protocol.

\section{Case 2}

Patient was reviewed every week and was continued with medical treatment (tab Medrol and tab zincovit ) as well as physiotherapy exercises.

\section{Discussion}

SARS-CoV-2 is an enveloped, non-segmented, singlestranded, positive-sense RNA virus that belongs to the beta-coronaviridae family.

Direct damage to specific receptors, cytokine-related injury, secondary hypoxia, and retrograde travel along nerve fibers are among routes by which SARS-CoV-2 causes neurologic damage.

Red cells distribution width was more increased in COVIDassociated Guillain-Barré syndrome. Also, lymphocytes and monocytes more in COVID. Table 1 was taken from nerve conduction velocity reports of each patient.

Table I.Comparison of NCV in COVID Positive and Negative GBS Patient

\begin{tabular}{|c|c|c|}
\hline \multicolumn{2}{|c|}{ COVID Positive } & COVID Negative \\
\hline $\begin{array}{c}\text { Rt. Median } \\
\text { Latency }\end{array}$ & Ampiltude 0 & $\begin{array}{c}\text { Rt. Median } \\
\text { L - Nr A - Nr }\end{array}$ \\
\hline \multicolumn{2}{|c|}{ Rt. Ulnar } & $\begin{array}{c}\text { Rt. Ulnar } \\
\text { L 1.69 A 13.4 }\end{array}$ \\
\hline \multicolumn{2}{|c|}{ Sural } \\
Latency - 2.9 Rt & Rt . Sural \\
& A 10.29 A 15.9 \\
\hline
\end{tabular}

The time between the onset of viral disease and the commencement of Guillain-Barré syndrome symptoms is similar to onset of Guillain-Barré syndrome during or after other infections.

Although various infectious agents have been linked to Guillain-Barré syndrome, infection with Campylobacter jejuni or Epstein-Barr virus may be a risk factor. ${ }^{6,7}$

It is impossible to say whether severe impairments and axonal involvement are typical symptoms of COVID-19 associated Guillain-Barré syndrome based on this observational case study involving two patients. We were unable to evaluate the impact of lower vital capacity due to Guillain-Barré syndrome-related neuromuscular failure in these individuals, but such an effect should be addressed if chest imaging findings are not comparable with the degree of respiratory insufficiency. Critical illness neuropathy and myopathy, which emerge later in the course of critical illness than Guillain-Barré syndrome, should be recognised from Guillain-Barré syndrome using COVID-19.

\section{Conclusion}

Recovery rate was much faster in non-COVID associated Guillain-Barré syndrome in comparison to COVID associated Guillain-Barré syndrome which was quite expected as there was single pathology to handle.

\section{Conflict of Interest: None}

\section{References}

1. World Health Organization[Internet]. Coronavirus Disease (COVID-19): Situation Report; 2020 (172) [cited $202120 \mathrm{Apr}$ ]. Available from: https://www.who. int/emergencies/diseases/novel-coronavirus-2019/ situation-reports.

2. Carrillo-Larco RM, Altez-Fernandez C, Ravaglia S, Vizcarra J. COVID-19 and Guillain-Barre Syndrome: a systematic review of case Reports. Wellcome Open Res. 2020:5;107. [Pubmed] [Google Scholar]

3. Mao L, Jin H, Wang M, Hu Y, ChenS, He Q, Chang J, Hong C, Zhou Y, Wang D, Miao X, Li Y, Hu B. Neurologic manifestations of hospitalized patients with coronavirus disease 2019 in Wuhan, China. JAMA Neurol. 2020:77(6);1-9. [Pubmed] [Google Scholar]

4. Piccione EA, Salame K, Katirji B. Guillain-Barré syndrome and related disorders neuromuscular disorders in clinical practice. Springer: New York, NY; 2002;573603. [Google Scholar]

5. Bridwell R, Long B, Gottlieb M. Neurologic complications of COVID-19. Am J Emerg Med. 2020;38(7):1549.e3-e7. [Pubmed] [Google Scholar]

6. Kim JE, Heo JH, Kim HO, Song SH, Park SS, Park TH, Ahn JY, Kim MK, Choi JP. Neurological complications during treatment of Middle East respiratory syndrome. J Clin Neurol. 2017;13:227-233. [Pubmed] [Google Scholar]

7. Sharma K, TengsupakulS, Sanchez O, Phaltas R, Maertens P. Guillain-Barré syndrome with unilateral peripheral facial and bulbar palsy in a child: a case report. SAGE Open Med Case Rep 2019;7:2050313X19838750. [Pubmed] [Google Scholar] 